\title{
Invisibles y ciudadanía global en la formación del profesorado de Educación Secundaria
}

\section{Invisibles and global citizenship in secondary education teacher training}

\author{
Maria Ballbé Martínez \\ Gustavo González Valencia \\ Universitat Autònoma de Barcelona \\ Delfín Ortega-Sánchez \\ Universidad de Burgos
}

\begin{abstract}
Resumen
El objetivo del presente estudio fue analizar qué piensa y cómo reflexiona el futuro profesorado de Educación Secundaria $(n=61)$ sobre problemas y conflictos sociales controvertidos, que afectan de manera local/global la vida de las personas, y que se pueden apreciar en el tiempo, el espacio geográfico, a través de las interrelaciones sociales, etc. La investigación fue de carácter mixto. La información se obtuvo mediante un cuestionario que planteaba el análisis de un tema controvertido. El análisis de la información se hizo siguiendo los principios metodológicos del análisis de contenido cuantitativo. Los resultados muestran que el futuro profesorado no alcanza un nivel crítico de literacidad, circunstancia que subraya la necesidad de proporcionar espacios para la enseñanza y la discusión de asuntos controvertidos con alcance global.
\end{abstract}

Palabras clave: Estudios sociales; Temas controvertidos; Perspectiva crítica; Formación del profesorado; Globalización

\section{\&}

\begin{abstract}
The aim of this study is to analyze what future Secondary Education teachers think $(n=61)$ and how they reflect on controversial social problems and conflicts affecting local/global issues, rethinking historical time, geographical space and social interrelations in the world. It was a mixed method research study. The information was obtained through a questionnaire that proposed the analysis of a controversial issue. The information analysis was done following the methodological principles of quantitative content analysis. The results show that future teachers do not reach a satisfactory critical level of literacy, a circumstance that underlines the need for spaces for teaching and discussion about controversial issues with a global significance.
\end{abstract}

Keywords: Social studies; Controversial issues;

Critical perspective; Teacher education;

Globalization 


\section{INTRODUCCIÓN}

La enseñanza-aprendizaje de las ciencias sociales debe estar al servicio de una educación para la equidad, la libertad y la participación, es decir, en pro de la justicia social. A tal fin, es necesario desarrollar el pensamiento crítico y creativo de las personas. La situación actual, en un mundo globalizado, exige que el profesorado se plantee cambios en lo que enseña y para qué enseña historia o geografía, que tenga en cuenta, por ejemplo, la diversidad en la temporalidad histórica y disponga de una visión del territorio en el que viva que nos permita establecer las conexiones necesarias de interdependencia entre lo local y lo global.

Lo anterior nos exige investigar en la formación inicial del futuro profesorado de historia, geografía y ciencias sociales para contribuir a la educación de una ciudadanía global crítica (ECGC), y para la defensa de los derechos humanos y la justicia social global (Sant et al., 2015; Davies et al., 2018; Sant \& González, 2018; Santisteban \& González, 2013). La enseñanza de las ciencias sociales debe tener como propósito desarrollar una actitud crítica en la ciudadanía que cuestione las informaciones, que compruebe la fiabilidad de los medios, capaz de identificar las injusticias y renovar la esperanza en la democracia. Para esto, es necesario visibilizar la exclusión social que sufrían y sufren las personas, por ejemplo, los más pobres.

Las preguntas que guiaron nuestra investigación fueron las siguientes: ¿Nuestro futuro profesorado de Educación Secundaria es capaz de interpretar información donde se invisibiliza, se oculta o se niega la presencia de determinados grupos o colectivos sociales?, ¿los y las estudiantes de profesorado realizan una interpretación crítica de la información, generada por los medios de comunicación, desde los principios de una educación para la ciudadanía global (ECG)? Nuestra intención fue analizar qué piensa el futuro profesorado y cómo reflexiona sobre problemas y conflictos sociales controvertidos que afectan de manera local/global. El estudio tuvo como supuesto que el profesorado establecía relaciones entre las dimensiones teóricas de la ECG y el análisis de problemas que implican una conexión entre la escala local y global. En términos metodológicos se hizo una aproximación descriptiva-interpretativa.

\section{LA CIUDADANÍA EN EL CONTEXTO DE LA GLOBALIZACIÓN}

Kerr (1999), hace dos décadas, señaló que asistíamos a cambios globales sin precedentes, que planteaban desafíos a los Estados, las sociedades, los sistemas educativos, y la forma de comprender y concretar la educación para la ciudadanía. 
Después de 20 años, estos desafíos se han hecho realidad y están provocando impactos significativos a nivel local y global. Asimismo, han emergido o adquirido mayor visibilidad cuestiones de especial relevancia social y ambiental, como el cambio climático, el surgimiento de instituciones supranacionales, el crecimiento de los populismos, nuevos actores económicos a escala global, la influencia de las redes sociales virtuales, la presencia creciente de la inteligencia artificial en la vida cotidiana, y en definitiva los rápidos cambios sociales y el crecimiento de la desigualdad social en el contexto de la globalización entre otros. Todo ello convierte la profundización de la democracia y, como tal, la formación de ciudadanos críticos en una necesidad acuciante.

El avance del proceso de globalización ha mostrado su parte menos positiva en las últimas tres décadas, debido a la excesiva centralidad de la dimensión económica en detrimento de la política. Esta circunstancia ha generado el aumento de la pobreza, la desigualdad y la contaminación en el mundo. Lo anterior tiene su correlato, que se puede entender como positivo, en el surgimiento y crecimiento de organizaciones internacionales como Save the Children, Oxfam intermón, Amnistía Internacional, Human Right Watch, Transparencia Internacional, Greenpeace, etc., que se pueden entender como formas de materialización de la ciudadanía global, y que abren a los ciudadanos nuevas posibilidades de acción global. Estas instituciones muestran la conexión entre ciudadanía y globalización, bajo la categoría de ciudadanía global.

La globalización ha ejercido influencia en la manera en que las personas perciben el rol de los Estados nacionales en el contexto local y global, provocando, en ocasiones, que dicha relación se vaya diluyendo. En efecto, bajo la influencia de las instituciones supranacionales, los Estados ceden competencias que tienen un rol relevante en la vida de la ciudadanía (Rodrik, 2011), como las políticas fiscales y migratorias, la regulación del mercado laboral, las edades de jubilación o los sistemas educativos. Esto se asocia con un discurso hegemónico que se caracteriza por promover la promesa de un futuro mejor (Bauman, 2001), pero como sugirieron George y Wolf (2002), sólo ha generado un crecimiento de la exclusión y la desigualdad, y ha difuminado el rol del ciudadano.

Para la ciudadanía, este hecho tiene un doble efecto: parece que el papel del Estado se disuelve, porque lo deja todo al libre mercado (García Canclini, 2001); y por otro lado, se generan nuevas oportunidades y escenarios donde hacer ejercicio de su ciudadanía, porque se añade una nueva escala de acción social, como es la global. En este contexto, la ECG aparece como una necesidad y una urgencia, si se 
pretende construir un mundo con una mayor justicia social global que permita erradicar la pobreza y mejorar las posibilidades de vida de las personas. No sólo se trata de desafiar injustas estructuras sociales, sino de construir comunidades globales que requieren un compromiso con concepciones más amplias e inclusivas de la ciudadanía (Yemini et al., 2019), capaces de vincular la experiencia local con las transformaciones sociales (Westheimer \& Kahne, 2004).

\section{LA EDUCACIÓN PARA LA CIUDADANÍA GLOBAL (ECG)}

La ciudadanía es una construcción social con pasado, presente y futuro. En una perspectiva clásica, se asociaba a los Estados nacionales a los que pertenecían o se adscribían los ciudadanos y las ciudadanas. Lo anterior se reducía a la posesión de un documento de identificación, lo que significaba asumir un estatus jurídico, arraigado a lo local y a la identidad nacional. En la actualidad, la manera de entender la construcción de las identidades nacionales se aleja de una idea monolítica y homogénea de nación y, por el contrario, reconoce la diversidad como un aspecto esencial.

La ciudadanía, entendida en relación con una comunidad e identificada con un lugar (el Estado-Nación), ha sido permeada de manera significativa por la globalización y la posmodernidad. Por lo tanto, su formación debe ser revisada con la misma lógica, lo que nos lleva a plantearnos la noción de ECG (Andreotti, 2006; Davies, 2006; Shultz, 2007; Torres, 2015; Veugelers, 2010; Zahabioun et al., 2013).

El concepto de "ECG" provoca una serie de preguntas acerca del significado de la "ciudadanía", de la "globalización", del papel de la educación en una sociedad global y del sistema político global (Rodrik, 2011), entre otras. De acuerdo con Tully (2014), la combinación de "globalización" y "ciudadanía" en la "ciudadanía global" aglutina los significados de cada concepto, haciendo posible que emerjan nuevas y complejas maneras de entenderla.

La ciudadanía global crítica se define como la conciencia, el cuidado y la aceptación de la diversidad cultural, a la vez que promueve la justicia social y la sostenibilidad, aparejada con un sentido de responsabilidad de acción para transformar las estructuras sociales que provocan la desigualdad. Nuestras investigaciones previas (González, Ballbé \& Ortega-Sánchez, 2020) sugieren que la conciencia ciudadana de conexión con otras personas del mundo (conciencia global), y de su inserción en entornos en que se valora la ciudadanía global (entorno normativo), conduce a una mayor identificación como ciudadanos y ciudadanas globales. 
Cuando esta identidad global se yuxtapone con personas de otras realidades sociales e históricas (González, Castellví \& Massip, 2020), es más fácil la construcción de escenarios cada vez más democráticos y justos, a escala global (Sant \& González, 2018).

En la perspectiva planteada, la ECG se entiende como un tipo de educación que busca la formación de ciudadanos y ciudadanas que reconozcan, comprendan y se encuentren en la disposición de pensar y actuar globalmente en la búsqueda de justicia social. Para Lilley, Barker y Harris (2017) es "to explore forms of education that enable learners to address local and global challenges, as socially responsible, critical and ethical thinking graduates, a disposition consistent with the global citizen" (p. 7). Esta perspectiva trasciende las figuras del Estado-nación, y se interesa por la búsqueda de los más altos imperativos morales y por la construcción de justicia social global. Desde una perspectiva crítica, la ECG busca la formación de ciudadanos y ciudadanas que participen en la construcción de una comunidad política (Cortina, 2003) "que conoce y se preocupa por asuntos contemporáneos en todo el mundo" (Dunn, 2002, p. 10).

\section{LA FORMACIÓN DEL PROFESORADO Y LA ECG}

Son múltiples las investigaciones acerca de la ECG, pero pocas las centradas en la formación del profesorado. En la revisión de la literatura, se encontraron estudios que indagaron sobre los discursos sobre la ECG de los futuros y futuras maestras (Yemini, 2017; Kim, 2019), la influencia de la educación global para asumirse como ciudadanos y ciudadanas globales (Larsen \& Searle, 2017), el profesorado en activo (Goren \& Yemini, 2016, 2017a; Goren et al., 2019; Çolak et al., 2019; Leduc, 2013), las finalidades de su enseñanza (Bruce et al., 2019), la valoración de propuestas o metodologías para la enseñanza (O’Meara et al., 2018), y la eficacia de los programas en la formación del profesorado para formar en ciudadanía global (Kopish 2016; Yang et al., 2017). Estas investigaciones coinciden en destacar la necesidad de incluir la ECG en la formación del profesorado.

Kim (2019) mostró que los programas de formación del profesorado ofrecen pocos espacios para enseñar y discutir sobre la ECG y sus implicaciones para la enseñanza. Planteamientos similares fueron el punto de partida del trabajo de Tarozzi e Inguaggiato (2019), quienes realizaron una investigación acerca de la ECG en tres programas de formación del profesorado en igual número de países (Italia, Austria y República Checa). En este trabajo se encontró que las maneras de entender y enseñar la ECG responden a las realidades culturales y políticas de cada 
país, y que se pueden concretar en tres tipologías: centradas en los contenidos, centradas en los valores y centradas en las competencias.

En este sentido, la ECG sirve para que las personas puedan tener una mayor y mejor comprensión de la interrelación entre ciudadanía, política, democracia y proceso de globalización, para así comprender las implicaciones políticas, económicas, culturales, sociales y ambientales (O’Meara et al., 2018; Bruce et al., 2019). Igualmente, la investigación se identificó con lo propuesto por Goren \& Yemini (2017b), Tarozzi y Inguaggiato (2018), y UNESCO (2015) cuando plantean que la formación del profesorado es una fase crucial para tener una adecuada implementación de la ECG (Goren \& Yemini, 2017b).

\section{ENSEÑANZA DE LAS CIENCIAS SOCIALES Y EDUCACIÓN PARA LA CiUdadanía Global Crítica (ECGC)}

Desde la didáctica de las ciencias sociales, investigamos para responder a preguntas como ¿qué pueden aportar los estudios sociales a la solución de los problemas sociales que cada día son más globales y afectan de manera diferenciada a la ciudadanía? En una perspectiva basada en la ECG, cada una de las ciencias sociales debe revisarse y reinterpretarse para su enseñanza, desde los contenidos hasta las estrategias o la metodología. Lo anterior establece una relación directa con la manera de entender la educación, la enseñanza de las ciencias sociales y la ECG desde un enfoque crítico (Giroux, 2013) en tiempos de globalización (González, Castellví \& Massip, 2020).

La enseñanza de las ciencias sociales debe estar orientada al desarrollo del pensamiento social y cumplir una labor contra-socializadora (Benejam, 2002) o, en otras palabras, que permita develar los esquemas establecidos, identificar el trasfondo ideológico de cada acción social, para alcanzar una mayor justicia y transformación social (Pagès \& Santisteban, 2011).

Enseñar ciencias sociales, en particular, la historia y la geografía desde una perspectiva crítica, contribuye a que comprendamos y participemos en la solución de las problemáticas que enfrentamos como sociedad, cada día más globales. El conjunto de las ciencias sociales nos aporta una comprensión más amplia, profunda y global de los hechos sociales y, en concreto, de la relación entre ciudadanía y globalización.

La globalización ha alentado a muchas disciplinas a examinar la naturaleza de ciudadanía y de la identidad y, de manera más general, los efectos del aumento 
de la interconexión con otros. Uno de sus resultados es la posibilidad de identificarse con el mundo. Por lo tanto, un modelo para entender la ECG debe considerar esta diversidad de contribuciones. En este sentido, la enseñanza de las ciencias sociales y la ECG, en el actual contexto social, político y educativo, se convierte en un campo en disputa, que precisa de un marco integrador de estas ciencias y de los aportes que pueden realizar en la comprensión y solución de los problemas sociales globales (Pagès \& Santisteban, 2014, Andreotti, 2006; Davies, 2006; Shultz, 2007; Torres, 2015, Veugelers, 2010; Zahabioun et al., 2013).

En esta línea, la presente investigación tomó como punto de partida la definición de ECGC, planteada por Oxley y Morris (2013) y por otros investigadores (Andreotti, 2006; Shultz, 2007), con el fin de elaborar una propuesta que reflejase las potenciales contribuciones de las ciencias sociales y humanas (Figura 1) con mayor presencia discursiva en el currículo (Real Decreto 1105/2014).

Figura 1. Dimensiones de ECGC. Fuente: González, Ballbé y Ortega (2020)

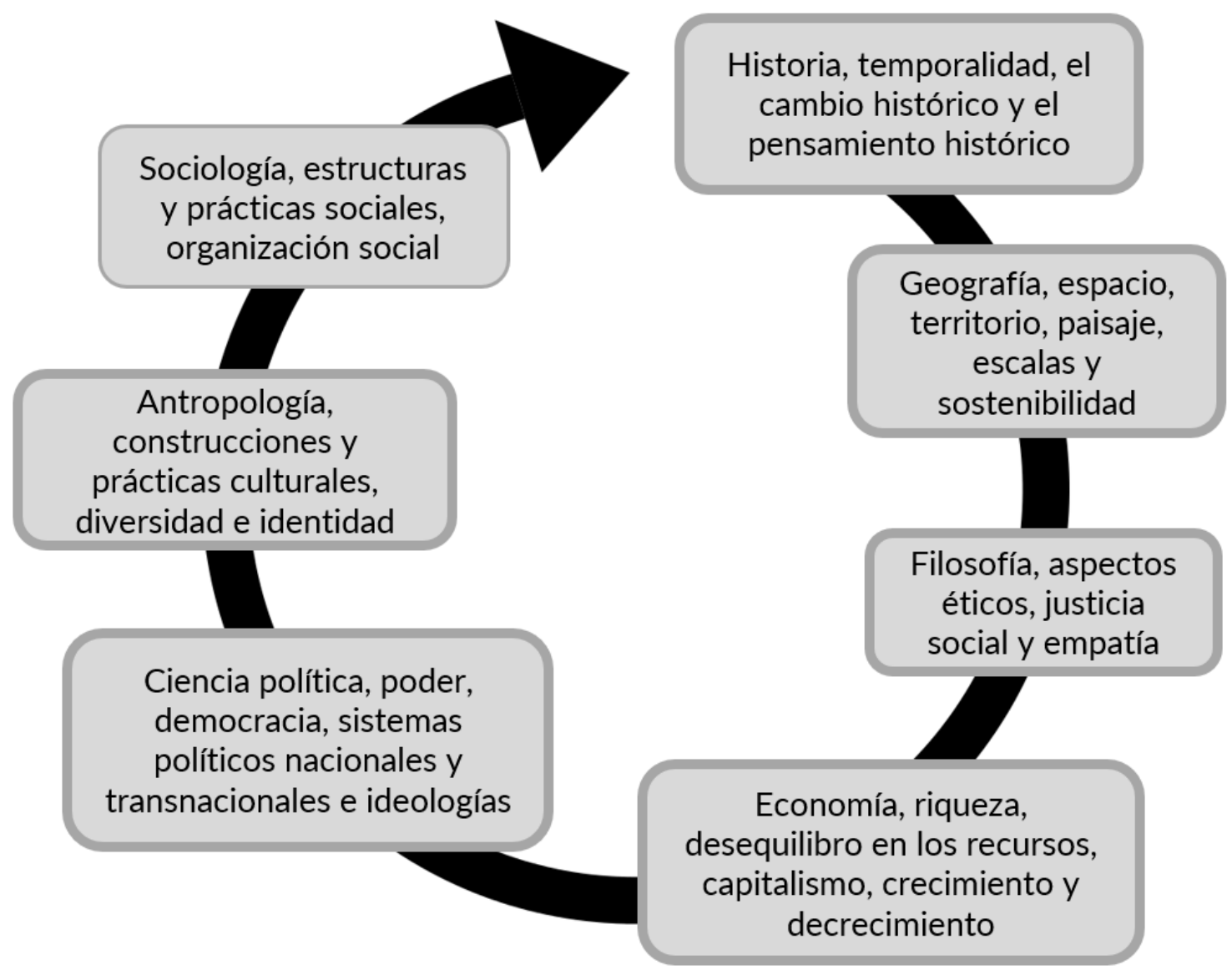


Esta manera de entender la interacción entre la ECG y el currículo proporcionó un modelo para el análisis de los datos. Éste se propuso a partir del supuesto de que los hechos sociales se analizan y explican desde la interrelación de diversas ciencias o disciplinas (O’Meara et al., 2018). Lo anterior hizo que el equipo de investigación pudiera identificar la presencia/ausencia de la dimensión global en los discursos del profesorado en formación y dar respuesta a las preguntas de investigación planteadas.

\section{MATERIAL Y MÉTODO}

El estudio se posiciona en los principios metodológicos descriptivo-interpretativos. Para el análisis de los datos obtenidos, se aplicaron los principios del análisis de contenido cuantitativo, a través del análisis estadístico descriptivo, de las prácticas de literacidad evidenciadas por el futuro profesorado, y de la aparición-ausencia y densidad textual de las dimensiones propuestas en el modelo teórico (Krippendorf, 1990). La finalidad de la investigación no fue medir, sino comprender cómo el futuro profesorado refleja el concepto de ECG y las dimensiones teóricas planteadas (temporalidad de los hechos; aspectos económicos; democracia, política e ideología; espacio/s de los hechos; ética y justicia social; prácticas culturales y estructuras sociales).

Los datos textuales fueron transcritos de forma fiel a como fueron obtenidos, organizados en una matriz para su análisis, y codificados temáticamente (Flick, 2012). El análisis de los datos se realizó a partir de tablas de frecuencias absolutas y relativas, relacionadas con tres niveles de literacidad textual-digital (Castellví et al., 2019; Castellví et al., en prensa) (nivel 1: descripción; nivel 2: análisis y relación; nivel 3: interpretación, relación y propuesta de acción social), y con las dimensiones explicativas de la ECGC propuesta: temporalidad de los hechos, aspectos económicos, democracia, política e ideología, espacio/s de los hechos, ética y justicia social, prácticas culturales y estructuras sociales. La presencia de un mayor número de códigos (dimensiones de la ECG) es indicador, en consecuencia, de la capacidad del futuro profesorado para analizar casos con una perspectiva interdependiente, compleja y crítica. 
Tabla 1. Niveles de literacidad crítica textual digital

(Castellví et al., 2019; Castellví et al., en prensa)

\begin{tabular}{ll}
\hline N1 & $\begin{array}{l}\text { Proporciona información descriptiva de la que aparece en la noticia } \\
\text { en cualquiera de las dimensiones explicativas de la ECGC: dimensio- } \\
\text { nes: temporalidad de los hechos, aspectos económicos, democracia, po- } \\
\text { lítica e ideología, espacio/s de los hechos, ética y justicia social, prácti- } \\
\text { cas culturales y estructuras sociales }\end{array}$ \\
\hline N2 & $\begin{array}{l}\text { Analiza la información acerca del hecho que aparece en la noticia y } \\
\text { establece relaciones entre algunas de las dimensiones explicativas } \\
\text { de la ECGC: dimensiones: temporalidad de los hechos, aspectos econó- } \\
\text { micos, democracia, política e ideología, espacio/s de los hechos, ética y } \\
\text { justicia social, prácticas culturales y estructuras sociales }\end{array}$ \\
\hline N3álisis y relación & $\begin{array}{l}\text { Interpreta la información acerca del hecho que aparece en la noticia } \\
\text { Interpretación, relación } \\
\text { y propuesta de acción } \\
\text { social }\end{array}$ \\
$\begin{array}{l}\text { de la ECGC: dimensiones: temporalidad de los hechos, aspectos econó- } \\
\text { micos, democracia, política e ideología, espacio/s de los hechos, ética y } \\
\text { justicia social, prácticas culturales y estructuras sociales. Toma posi- } \\
\text { ción crítica desde la que propone acciones de cambio. Propone ac- } \\
\text { ciones orientadas a la construcción de justicia social. }\end{array}$ \\
\hline
\end{tabular}

\section{PARTICIPANTES}

La información se obtuvo de cuatro grupos de futuro profesorado de Educación Secundaria y Bachillerato $(n=61)$, matriculado en tres universidades públicas en el curso académico 2018-2019 ( $\mathrm{U}_{1}=28,47,4 \%$; $\mathrm{U}_{2}=25,42,3 \%$; $\left.\mathrm{U}_{3}=8,13,5 \%\right)$. No se tuvo acceso a la información de género porque fue un ítem que no se debía contestar de manera obligatoria. Las universidades donde se obtuvo la información participan de un proyecto competitivo denominado Enseñar y aprender a interpretar problemas y conflictos contemporáneos. ¿Qué aportan las ciencias sociales a la formación de una ciudadanía global crítica? (EDU2016 80145-P). Las personas que contestaron los cuestionarios fueron estudiantes del Máster en Educación Secundaria. El muestreo fue por conveniencia, debido a la facilidad de acceso a la población. La respuesta al cuestionario fue voluntaria.

En todo momento, se garantizó el anonimato de la identidad de los y las participantes, y de los datos obtenidos. Con este fin, los nombres utilizados en este estudio no se corresponden con las identidades del alumnado

\section{INSTRUMENTO}

La obtención de la información se realizó mediante cuestionario (González, 2010). Éste fue construido a partir del marco teórico. En éste se plantean a los y las 
estudiantes una serie de casos, definidos como temas controvertidos (Santisteban, 2019), sobre los que se ha de expresar una opinión o representación.

En el caso central de esta investigación, se analiza la capacidad de interpretar críticamente una noticia sobre la pobreza y la desigualdad social, acompañada de un anuncio de unos grandes almacenes en el que una modelo promociona rebajas en la ropa de moda. Esta técnica publicitaria que tiene como objetivo promover el consumo de determinados productos es común en los periódicos y otros medios digitales, pero éticamente no aceptable, ya que invisibiliza la desigualdad y banaliza la pobreza.

Figura 2. Caso central. El riesgo de la pobreza, en un nivel de alarma. Fuente: el Periódico

\section{日 riesgo de pobreza, en un nivel de alarma}

Que el $22,3 \%$ de los ciudadanos estén en riesgo de pobreza debería encender las alarmas de los poderes públicos

$N$ эnes 25/0<20 $\cdots$ Actualizado el 26/04/2017 a as 17:30 CEST

$000 \div$

Esta vez no es una oenegé o una ertidad dedicada a asistir a los más necesitados, sino un organismo oficial: el Instituto Nacional de Estadística ha certificado los niveles alarmantes de vulnerabilidad que sufre una parte importante de la población española como consecuencia de una crisis devastadora de cuyos efeetos no se ve el final.

El dato de que el $22,3 \%$ de los ciudadanos se encuentran en riesgo de pobreza es de una contundencia que debería encender las alarmas de los poderes públicos y espolearles a combatir con

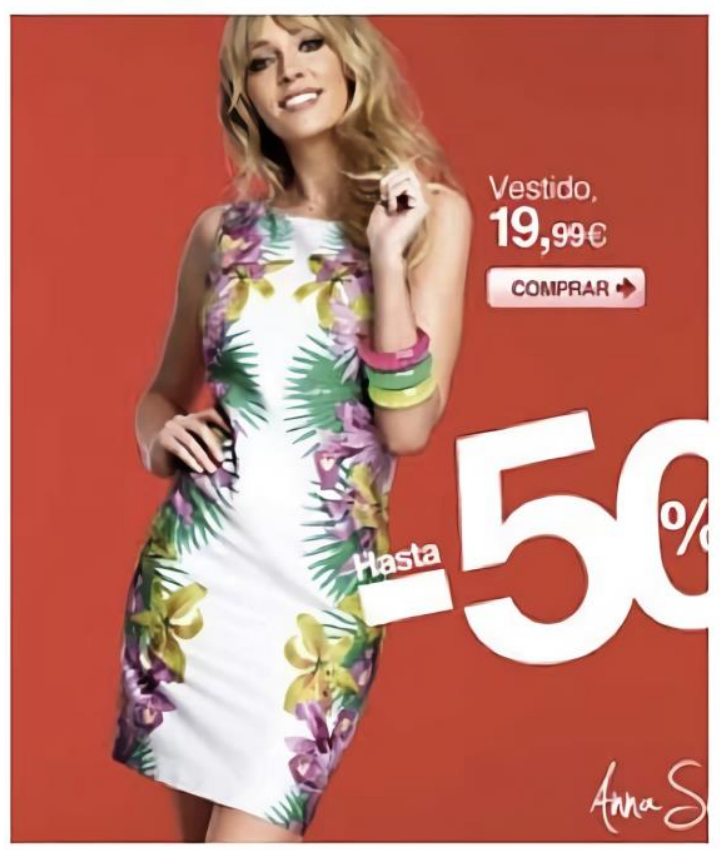

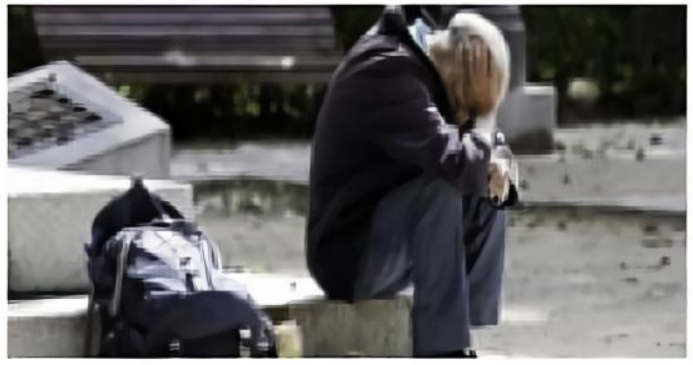

medidas eficaces el agrandamiento de la brecha social. Hoy los ricos son más ricos que antes de la crisis, y los pobres son más pobres, pero sobre todo amplísimas capas de la población, las clases medias y bajas, han descendido uno o varios escalones en su calidad de vida y -lo que es más hiriente- no ven la posibilidad de recuperar el terreno perdido. España es más desigual que hace una década. Que en tomo al $40 \%$ de los hogares no tengan capacidad para afrontar gastos imprevistos o no puedan irse de vacaciones ni una semana al año son dos expresiones de esa grave patología social.

EI INE también pone cifras a la educación como factor de protección en situaciones de crisis: el riesgo de pobreza es tres veces superior entre quienes solo tienen estudios básicos. $\mathrm{Si}$ esa obviedad sirviera para que la Administración hiciera una apuesta estratégica por la enseñanza como fac.or de riqueza del país, habría motivos para la esperanza a medio plazo, pero no parece ser el caso. 
La imagen iba acompañada de la siguiente pregunta: ¿Qué información te ofrece la página anterior del diario? Valora el contenido de toda la página, incluidos el texto y todas las imágenes. La composición de la página refleja un tema controvertido relacionado con el impacto de la globalización en la vida cotidiana, que puede ser analizado bajo las dimensiones propuestas y permite reflejar diferentes niveles de literacidad crítica.

\section{RESUlTADOS}

Los resultados obtenidos en las universidades participantes son similares, a pesar de la diferencia en el tamaño de las muestras. Los datos revelan que el nivel de literacidad con más presencia en los textos corresponde al análisis y la relación $(f=32,54 \%)$ (Tabla 2$)$.

Tabla 2. Niveles de literacidad crítica

\begin{tabular}{lcccccccc}
\hline \multirow{2}{*}{$\begin{array}{l}\text { Niveles de } \\
\text { literacidad crítica }\end{array}$} & \multicolumn{2}{c}{ U1 } & \multicolumn{2}{c}{ U2 } & \multicolumn{2}{c}{ U3 } & \multicolumn{2}{c}{ Total } \\
\cline { 2 - 9 } & 12 & $43 \%$ & 11 & $44 \%$ & 0 & $0 \%$ & 23 & $39 \%$ \\
\hline N1 Descripción & 12 & $46 \%$ & 13 & $52 \%$ & 6 & $100 \%$ & 32 & $54 \%$ \\
$\begin{array}{l}\text { N2 Análisis y } \\
\text { relación }\end{array}$ & 13 & & & & & & & \\
$\begin{array}{l}\text { N3 Interpretación, } \\
\text { relación y } \\
\text { propuesta de } \\
\text { acción social }\end{array}$ & 3 & $11 \%$ & 1 & $4 \%$ & 0 & $0 \%$ & 4 & $7 \%$ \\
\hline$N$ & 28 & $100 \%$ & 25 & $100 \%$ & 6 & $100 \%$ & 59 & $100 \%$ \\
\hline$N$
\end{tabular}

A continuación, presentamos algunos de los comentarios escritos de los participantes (numerados y con un pseudónimo que preserva su género). Un poco más de la mitad de los futuros profesores profesoras demuestran capacidad para situarse en un nivel de análisis y relación (N2): analizar la noticia, identificando los mecanismos persuasivos de los medios de comunicación a la hora de utilizar la composición del formato, de los textos y de las imágenes para construir relatos hegemónicos. Asimismo, son capaces de elaborar un texto con argumentos propios sobre el problema social planteado (la pobreza). Sus argumentos se fundamentan en los valores democráticos, los derechos humanos y la justicia social:

(1) Laura: La [imagen] situada en la parte superior derecha nos muestra una visión de la pobreza que no se corresponde con la realidad, es decir, se puede ser pobre teniendo incluso trabajo. La ubicada en la zona inferior izquierda es un anuncio del Corte Inglés, donde 
vemos a una conocida presentadora anunciando las rebajas. Dos imágenes que están cargadas con el cinismo de la modernidad.

El segundo lugar lo ocupa el primer nivel de literacidad de descripción (N1) $(f=23$, un $39 \%)$. Las respuestas categorizadas en este nivel se centran en la descripción de los hechos y, destacan las contradicciones entre los elementos compositivos de la página sin analizar las razones del problema social propuesto:

(2) Sergio: El artículo habla sobre el riesgo de pobreza en España (...) [y]concluye haciendo un llamamiento a mejorar la educación, pues reclama que el riesgo de caer en la pobreza es tres veces mayor en personas sin estudios. Por tanto, declara que para evitar la creciente desigualdad es necesario mejorar la enseñanza para que suponga la principal fuente de riqueza del país. [].

El nivel de literacidad de relación y propuesta de acción social (N3) registra la frecuencia más baja $(f=4$, un $7 \%$ ). El relato de Marta se corresponde con este nivel y refleja el interés por la transformación social a favor de una sociedad más justa y equitativa. Con este fin, plantea posibles acciones sociales:

Deberíamos plantearnos un nuevo pacto o contrato social y un nuevo sistema que nos ampare mejor a todos, y sea más solidario. No deja de ser irónico y grotesco que, insertado en la noticia, haya un anuncio de unos grandes almacenes que nos empujan al consumismo, uno de los factores de declive de nuestro sistema social y económico, en mi opinión. [Marta].

Los resultados obtenidos sobre las dimensiones de la ECGC revelan que, en el conjunto de las respuestas, hay presencia de todas las dimensiones (Tabla 3).

Tabla 3. Dimensiones de la Educación para la Ciudadanía Global Crítica ECGC

\begin{tabular}{lcccccccc}
\hline \multirow{2}{*}{ Dimensiones } & \multicolumn{2}{c}{ U1 } & \multicolumn{2}{c}{ U2 } & \multicolumn{2}{c}{ U3 } & \multicolumn{2}{c}{ Total } \\
\cline { 2 - 9 } & $f_{i}$ & $h_{i}$ & $f_{i}$ & $h_{i}$ & $f_{i}$ & $h_{i}$ & $f_{i}$ & $h_{i}$ \\
\hline $\begin{array}{l}\text { Temporalidad de los } \\
\text { hechos }\end{array}$ & 1 & $1 \%$ & 3 & $5 \%$ & 0 & $0 \%$ & 4 & $3 \%$ \\
Aspectos económicos & 22 & $29 \%$ & 18 & $29 \%$ & 5 & $24 \%$ & 45 & $28 \%$ \\
$\begin{array}{l}\text { Democracia, política e } \\
\text { ideología }\end{array}$ & 13 & $17 \%$ & 13 & $21 \%$ & 6 & $29 \%$ & 32 & $20 \%$ \\
Espacio/s de los hechos & 4 & $5 \%$ & 3 & $5 \%$ & 0 & $0 \%$ & 7 & $4 \%$ \\
Ética y justicia social & 8 & $11 \%$ & 9 & $14 \%$ & 3 & $14 \%$ & 20 & $13 \%$ \\
Prácticas culturales & 3 & $4 \%$ & 2 & $3 \%$ & 1 & $5 \%$ & 6 & $4 \%$ \\
Estructuras sociales & 25 & $33 \%$ & 15 & $24 \%$ & 6 & $29 \%$ & 46 & $29 \%$ \\
\hline $\mathrm{N}$ & 76 & $100 \%$ & 63 & $100 \%$ & 21 & $100 \%$ & 160 & $100 \%$ \\
\hline
\end{tabular}


La dimensión con mayor incidencia corresponde a las estructuras sociales (29\%). Los datos no muestran diferencias entre las universidades, excepto en la dimensión estructuras sociales donde la U1 concentra una presencia más intensa. A esta dimensión le siguen los aspectos económicos (28\%), la democracia, la política y la ideología $(20 \%)$, y la ética y la justicia social $(13 \%)$. Son notables las escasas referencias a las dimensiones temporalidad (3\%) y espacio/s de los hechos (4\%), y prácticas culturales ( $4 \%)$.

En términos generales, las tres dimensiones con mayor presencia (estructuras sociales, aspectos económicos, y democracia, política e ideología) generan un triángulo narrativo en el relato del futuro profesorado, ubicado entre el segundo (análisis y relación) y el tercer nivel de literacidad (relación y propuesta de acción social). Los textos de los y las estudiantes incluidos en el tercer nivel de literacidad hacen referencia a aspectos económicos y sociales, y una mayoría alude a los relacionados con la democracia, la política, la ideología, la ética y la justicia social. Esto evidencia que las respuestas contienen una mayor densidad de códigos en los análisis, como demuestra el siguiente fragmento:

(3) Xavier: [Se trata de] una información parcial, políticamente de derechas, (...) [que habla de] la pobreza como una patología social. (...) Así se ilustra curiosamente con una imagen de las rebajas, un momento en que la clase obrera desproletarizada y engañada por el sistema, que ha dejado sus espaldas a disposición de estos, compra sin parar objetos inútiles (...).

\section{DISCUSIÓN}

Nuestra investigación toma como punto de partida la perspectiva teórica de la ECGC de Oxley y Morris (2013), que destaca el papel de la ciudadanía para identificar y actuar contra las injusticias. Para ello, es necesario reconocer las dimensiones ideológicas y hegemónicas de los hechos sociales para avanzar en la construcción de una justicia social global.

Los datos muestran relaciones entre las dimensiones de ECG y los niveles de literacidad. Esto puede apreciarse en el número de dimensiones presentes en el análisis del hecho social. El reducido número de textos categorizados en el nivel interpretación, relación y propuesta de acción social sugiere que existe una escasez de espacios para la enseñanza y la discusión sobre la ECG, con importantes implicaciones educativas (Kim, 2019; Tarozzi \& Mallon, 2019; Yang et al., 2017; Goren \& Yemini, 2017b). 
El profesorado en formación logra establecer relaciones entre la escala global y la local (Tarozzi \& Inguaggiato 2018, 2019; Goren \& Yemini, 2017b; UNESCO, 2015). Sin embargo, esta relación se reduce a la enunciación, casi anecdótica, de algunas dimensiones de la ECGC empleadas para la comprensión de los hechos sociales. En este marco, los y las estudiantes logran relacionar las consecuencias de la globalización con la pobreza, pero plantean pocas o nulas acciones para contrarrestar las consecuencias negativas de la globalización. En consecuencia, creemos que es necesario proporcionar herramientas para superar el análisis descriptivo de los hechos y alcanzar una verdadera ECGC, tal como apuntan Goren y Yemini (2017b).

Los resultados muestran que alrededor de la mitad del profesorado en formación se ubica en el nivel de literacidad análisis y relación. Esto coincide con la dimensión humanista liberal de Bruce et al. (2019), donde el análisis o la comprensión es una característica, sin la necesidad de llegar a acciones de transformación sobre lo que se comprende. Asimismo, el estudio muestra que el futuro profesorado no alcanza el nivel interpretación, relación y propuesta de acción social, es decir, a proponer acciones de mejora de la realidad social, hallazgo que difiere de los resultados de O’Meara et al. (2018). Estos autores consideran que el profesorado es más proactivo para la enseñanza de la ciudadanía global crítica cuando han sido formado en estos temas. Asimismo, ese hallazgo es consistente con los de Ortega-Sánchez y Pagès (2017), quienes se encontraron niveles similares de lectura de análisis de temas controvertidos.

La propuesta teórica de las dimensiones de la ECGC empleada para su análisis resultó oportuna, porque permitió conocer cómo el profesorado en formación analiza un hecho social. El 4\% de los textos categorizados en el nivel interpretación, relación y propuesta de acción social tienen en común la referencia a aspectos asociados a las dimensiones estructuras sociales, aspectos económicos, democracia, política, ideología, y ética y justicia social. Las acciones que emergieron estaban orientadas a combatir la injusticia social de la pobreza, habitualmente asociada a las políticas neoliberales y capitalistas.

Los datos muestran la ausencia de diferencias por universidad participante, indicador de que el futuro profesorado confiere un peso relevante a la dimensión global por encima de la local en el análisis de un problema social relevante. Estos resultados no representan la negación de la dimensión contextual del hecho, como confirman los hallazgos de Tarozzi e Inguaggiato (2019), y de González, Ballbé y 
Ortega-Sánchez (2020), es decir, las características del contexto influyen en la manera como se entiende la dimensión global de un problema social relevante.

\section{CONCLUSIONES}

Los hallazgos nos indican que el profesorado en formación se encuentra algo distante en la aplicación de una ECGC orientada a la justicia social. Faltan propuestas que les permitan comprender las implicaciones de la globalización, con el fin de alcanzar niveles de análisis de los hechos dirigidos a la búsqueda de la justicia social. El uso de estrategias que favorezcan la interrelaciones entre la globalización y los hechos sociales -a través del análisis de casos o incluso del aprendizaje serviciosería una buena forma de promover una mayor comprensión de la ECGC (Kopish, 2016).

Si el profesorado en formación tiene una comprensión parcial sobre las consecuencias de la globalización y sobre cómo actuar ante los retos que presenta, difícilmente la ECGC llegará a las aulas. Los programas de formación inicial y continua del profesorado deben considerar los aspectos históricos, geográficos, políticos, económicos, sociales y éticos en la búsqueda de la justicia social. Sólo de este modo se podrá pensar en un profesorado crítico y orientado a la transformación social, capaz de superar las consecuencias negativas, fundamentalmente económicas, de los actuales procesos de globalización (González, Ballbé \& OrtegaSánchez, 2020).

\section{REFERENCIAS}

Andreotti, V. (2006). Soft versus critical global citizenship education. Policy \& Practice-A Development, 3, 40-51.

Bauman, Z. (2001). La Globalización: consecuencias humanas. Fondo de Cultura Económica.

Benejam, P. (2002). Las finalidades de la educación social. En P. Benejam \& J. Pagès (Eds.), Enseñar y Aprender Ciencias Sociales, Geografía e Historia en la Educación Secundaria (pp. 33-52). Barcelona: Horsori.

Bruce, J., North, C., \& FitzPatrick, J. (2019). Preservice teachers' views of global citizenship and implications for global citizenship education. Globalisation, Societies and Education, 17(2), 161-176. https://doi.org/10.1080/14767724.2018.1558049

Castellví, J., Ballbé, M., \& Pagès, J. (en prensa). La literacidad crítica digital en el grado de educación primaria: un estudio con maestras y maestros en formación. En Repensar el currículum de Ciencias Sociales: prácticas educativas para una ciudadanía crítica. Universitat de València - AUPDCS.

Castellví, J., Massip, M., \& Pagès, J. (2019). Emociones y pensamiento crítico en la era digital: un estudio con alumnado de formación inicial. Revista de investigación en Didáctica de las Ciencias Sociales, 5, 23-41. 
Çolak, K., Kabapınar, Y., Öztürk, C., \& Öztürk, C. (2019). Social Studies Courses Teachers’ Views on Global Citizenship and Global Citizenship Education. Ted Eğitim Ve Bilim, 44(197). http://doi.org/10.15390/EB.2019.7721

Cortina, A. (2003). Ciudadanos del mundo: hacia una teoría de la ciudadanía. Alianza.

Davies, I., Ho, L-C., Kiwan, D., Peck, C., Peterson, A., Sant, E., \& Waghid, Y. (Eds.) (2018). The Palgrave handbook of global citizenship and education. Palgrave Macmillan.

Davies, L. (2006). Global citizenship: abstraction or framework for action? Educational Review, 58(1), 5-25. https://doi.org/10.1080/00131910500352523

Dunn, R. E. (2002). Growing good citizens with a world-centered curriculum. Educational Leadership, 60(2) 10-13.

Flick, U. (2012). Introducción a la investigación cualitativa. Morata.

García Canclini, N. (2001). La Globalización imaginada. Paidós.

George, S. \& Wolf, M. (2002). La globalización liberal: a favor y en contra. Anagrama.

Giroux, H. (2013). La pedagogía crítica en tiempos oscuros. Praxis Educativa, XVII(2), 13-26.

González, G. (2010). La transición entre teoría y campo de investigación en la didáctica de las ciencias sociales. En R. Ávila, P. Rivero \& P. Domínguez (Eds.), Metodología de investigación en Didáctica de las Ciencias Sociales. (pp. 23-35). a + d arte digital, S. L

González, G. Castellví, J., \& Massip, M. (2020). Heritage education and goblal citizenship education. En J. Cuenca \& E. Delgado, E. (Eds.), Handbook of Research on Citizenship and Heritage Education. (pp. 80-102). IGI Global.

González, G., Ballbé, M., \& Ortega-Sánchez, D. (2020). Global Citizenship and Analysis of Social Facts: Results of a Study with Pre-Service Teachers. Social Sciences, 9, 65-84. https://doi.org/10.3390/socsci9050065

Goren, H., \& Yemini, M. (2016). Global citizenship education in context: teacher perceptions at an international school and a local Israeli school. Compare: A Journal of Comparative and International Education, 46(5), 832-853. https://doi.org/10.1080/03057925.2015.1111752

Goren, H., \& Yemini, M. (2017a). The global citizenship education gap: Teacher perceptions of the relationship between global citizenship education and students' socio-economic status. Teaching and Teacher Education, 67, 9-22. https://doi.org/10.1016/J.TATE.2017.05.009

Goren, H., \& Yemini, M. (2017b). Global citizenship education redefined - A systematic review of empirical studies on global citizenship education. International Journal of Educational Research, 82, 170-183. https://doi.org/10.1016/J.IJER.2017.02.004

Goren, H., Maxwell, C., \& Yemini, M. (2019). Israeli teachers make sense of global citizenship education in a divided society-religion, marginalisation and economic globalisation. Comparative Education, 55, 243-63.

Kerr, D. (1999). Citizenship education: an international comparison. Qualifications and Curriculum Authority.

Kim, Y. (2019). Global citizenship education in South Korea: ideologies, inequalities, and teacher voices. Globalisation, Societies and Education, 17(2), 177-193.

https://doi.org/10.1080/14767724.2019.1642182

Kopish, M. (2016). Preparing globally competent teacher candidates through cross-cultural experiential learning. Journal of Social Studies Education Research, 7(2), 75-108.

Krippendorff, K. (1990). Metodología de análisis de contenido. Paidós.

Kymlicka, W., \& Norman, W. (1994). Return of the citizen: A survey of recent work on citizenship theory. Ethics, 104(2), 352-381. 
Larsen, M., \& Searle, M. (2017). International service learning and critical global citizenship: A cross-case study of a Canadian teacher education alternative practicum. Teaching and Teacher Education, 63, 196-205. https://doi.org/10.1016/j.tate.2016.12.011

Leduc, R. (2013). Global citizenship instruction through active participation: What is being learned about global citizenship? The Educational Forum, 77(4), 394-406. https://doi.org/10.1080/00131725.2013.822038

Lilley, K., Barker, M., \& Harris, N. (2017). The global citizen conceptualized. Journal of Studies in International Education, 21(1), 6-21. https://doi.org/10.1177/1028315316637354

O'Meara, J., Huber, T., \& Sanmiguel, E. (2018). The role of teacher educators in developing and disseminating global citizenship education strategies in and beyond US learning environments. Journal of Education for Teaching, 44(5), 556-573. https://doi.org/10.1080/02607476.2018.1516347

Ortega-Sánchez, D., \& Pagès, J. (2017). Literacidad crítica, invisibilidad social y género en la formación del profesorado de Educación Primaria. REIDICS: Revista de investigación en Didáctica de las Ciencias Sociales, 1, 102-117.

Oxley, L., \& Morris, P. (2013). Global citizenship: A typology for distinguishing its multiple conceptions. British Journal of Educational Studies, 61(3), 301-325.

Pagès, J., \& Santisteban, A. (2011). Les quëstions socialment vives i l'ensenyament de les ciències socials. Universitat Autònoma de Barcelona.

Pagès, J., \& Santisteban, A. (2014). Una mirada del pasado al futuro en la didáctica de las ciencias sociales. En J. Pagès, J. \& A. Santisteban (Eds.), Una mirada al pasado y un proyecto de futuro. Investigación e innovación en didáctica de las ciencias sociales (pp. 17-39). Servicio de Publicaciones de la UAB - AUPDCS.

Real Decreto 1105/2014, de 26 de diciembre, por el que se establece el currículo básico de la Educación Secundaria Obligatoria y del Bachillerato. Boletín Oficial del Estado, 3, de 3 de enero de 2015.

Rodrik, D. (2011). The globalization paradox: why global markets, states, and democracy can't coexist. Oxford University Press.

Sant, E. \& González, G. (2018). Global citizenship education in Latin America. In I. Davies, L. Ch. Ho, D. Kiwan, C.L. Peck, A. Peterson, E. Sant \& Y. Waghid (Eds.), The Palgrave handbook of global citizenship and education (pp. 67-82). Palgrave Macmillan.

Sant, E., Davies, I., \& Santisteban, A. (2015). Citizenship and identity: the self-image of secondary school students in England and Catalonia. British Journal of Educational Studies, 64(2), 235-260. https://doi.org/10.1080/00071005.2015.1070789

Santisteban, A. (2019). La enseñanza de las ciencias sociales a partir de problemas sociales o temas controvertidos: estado de la cuestión y resultados de una investigación. El Futuro del Pasado, 10, 57-79. https://doi.org/10.14516/fdp.2019.010.001.002

Santisteban, A., y González-Valencia, G. (2013). Sociedad de la información, democracia y formación del profesorado ¿qué lugar debe ocupar el pensamiento crítico? In J.J. Díaz, A. Santisteban \& A. Cascajero (Eds.), Medios de comunicación y pensamiento crítico: nuevas formas de interacción social (pp. 761-770). Editorial Universidad de Alcalá.

Shultz, L. (2007). Educating for global citizenship: Conflicting agendas and understandings. Alberta Journal of Educational Research, 53(3), 248-258.

Tarozzi, M. \& Inguaggiato, C. (2018). Implementing global citizenship education in EU primary schools: The role of government ministries. International Journal of Development Education and Global Learning, 10(1), 21-38. https://doi.org/10.18546/IJDEGL.10.1.03

Tarozzi, M. \& Inguaggiato, C. (Eds.) (2019). Teachers' education in GCE: emerging issues in a comparative perspective. Università di Bologna. 
Tarozzi, M. \& Mallon, B. (2019). 'Educating teachers towards global citizenship: A comparative study in four European countries'. London Review of Education, 17(2), 112-125.

https://doi.org/10.18546/LRE.17.2.02

Torres, C. (2015). Global Citizenship and Global Universities. The Age of Global Interdependence and Cosmopolitanism, 50(3), 262-279.

Tully, J. (2014). On global citizenship: James Tully in dialogue. Bloomsbury.

UNESCO. (2015). Global citizenship education: topics and learning objectives. UNESCO.

Veugelers, W. (2010). The moral and the political in global citizenship: Appreciating differences in education. Globalisation, Societies and Education, 9(3-4), 473-485.

Westheimer, J., \& Kahne, J. (2004). What kind of Citizen? The Politics of Educating for Democracy. American Educational Research Journal, 41(2), 237-269. https://doi.org/10.3102/00028312041002237

Yang, M., Kim, J., \& Kim, K. (2017). A Study on Variables Related to Global Citizenship Teaching Efficacy of Secondary School Pre-service Teachers and In-service Teachers. Association of Global Studies Education, 9(3), 77-104. https://doi.org/10.19037/agse.9.3.03

Yemini, M. (2017). Internationalization and global citizenship: policy and practice in education. Palgrave Macmillan.

Yemini, M., Tibbitts, F., \& Goren, H. (2019). Trends and caveats: Review of literature on global citizenship education in teacher training. Teaching and Teacher Education, 77, 77-89. https://doi.org/10.1016/J.TATE.2018.09.014

Zahabioun, S., Youssefy, A., Yarmohammadian, M. H., \& Keshtiaray, N. (2013). Global Citizenship Education and itsiImplications for curriculum goals at the age of globalization. International Education Studies, 6(1), 105-206.

\section{MARIA BALLBÉ MARTÍNEZ}

Profesora de secundaria y profesora asociada en la Facultad de Ciencias de la Educación de la Universitat Autònoma de Barcelona. Es miembro del Grup de Recerca en Didàctica de les Ciències Socials (GREDICS). Sus intereses de investigación son la enseñanza de las ciencias sociales, la renovación pedagógica y la formación inicial del profesorado.

maria.ballbe@uab.cat https://orcid.org/0000-0003-3882-1077

\section{GuSTAVO GONZÁLEZ VALENCIA}

Profesor lector (Serra Húnter) en la Faculta de Ciencias de la Educación de la Universidad Autónoma de Barcelona. Es miembro del Grup de Recerca en Didàctica de les Ciències Socials (GREDICS). Sus intereses investigativos son la educación ciudadana y democrática, la enseñanza de las ciencias sociales, la formación inicial del profesorado y la metodología de la investigación. 


\section{DELFÍN ORTEGA-SÁNCHEZ}

Profesor titular de la Universidad de Burgos. Director del Grupo de Investigación en Didáctica de la Historia y de las Ciencias Sociales (DHISO). Sus intereses investigadores se centran en la perspectiva de género y los problemas sociales en la enseñanza de la historia y en la formación inicial del profesorado.

dosanchez@ubu.es

Ballbé Martínez, M., González Valencia, G. \& Ortega-Sánchez, D. (2021). Invisibles y ciudadanía global en la formación del profesorado de Educación Secundaria. Bellaterra Journal of Teaching \& Learning Language \& Literature, 14(2), e910. https://doi.org/10.5565/rev/jt13.910

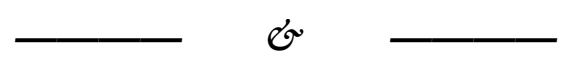

Rebut / Recibido / Received / Reçu: 25-06-2020

Acceptat / Aceptado / Accepted / Accepté: 21-07-2020 